TRANSACTIONS OF THE

AMERICAN MATHEMATICAL SOCIETY

Volume 363, Number 4, April 2011, Pages 1789-1804

S 0002-9947(2010)04844-5

Article electronically published on November 17, 2010

\title{
HODGE-LAPLACE EIGENVALUES OF CONVEX BODIES
}

\author{
ALESSANDRO SAVO
}

\begin{abstract}
We give upper and lower bounds of the first eigenvalue of the Hodge Laplacian acting on smooth $p$-forms on a convex Euclidean domain for the absolute and relative boundary conditions. In particular, for the absolute conditions we show that it behaves like the squared inverse of the $p$-th longest principal axis of the ellipsoid of maximal volume included in the domain (the John ellipsoid). Using John's theorem, we then give a spectral geometric interpretation of the bounds and relate the eigenvalues with the largest volume of a $p$-dimensional section of the domain.
\end{abstract}

\section{INTRODUCTION}

1.1. Notation. Let $M$ be a compact Riemannian manifold with smooth boundary, and let

$$
\Delta=d \delta+\delta d
$$

be the Hodge Laplacian acting on smooth differential $p$-forms on $M$. Here $d$ is the exterior differential and $\delta$ is the co-differential (the formal adjoint of $d$ with respect to the $L^{2}$-inner product of forms). We consider the eigenvalue problem for the absolute boundary conditions:

$$
\left\{\begin{array}{l}
\Delta \omega=\mu \omega \\
i_{N} \omega=0 \text { on } \partial M, \\
i_{N} d \omega=0 \text { on } \partial M,
\end{array}\right.
$$

where $\omega$ is a $p$-form, $N$ is the unit vector, normal to the boundary and pointing inward, and $i_{N}$ denotes interior multiplication. We denote by $\mu_{1}^{[p]}$ the first positive eigenvalue of the absolute problem (1.1).

The dual boundary conditions are the relative ones for which $J_{\partial M} \omega=J_{\partial M} \delta \omega=$ 0 , with $J_{\partial M}$ denoting restriction of a form to $\partial M$. We denote by $\lambda_{1}^{[p]}$ the first positive eigenvalue of the relative problem on $p$-forms. The Hodge $\star$ operator intertwines the two boundary conditions, and in particular

$$
\lambda_{1}^{[p]}=\mu_{1}^{[n-p]} .
$$

Received by the editors June 10, 2008.

2010 Mathematics Subject Classification. Primary 58J50.

Key words and phrases. Laplacian on forms, eigenvalues, convex bodies, John ellipsoid.

This work was partially supported by the COFIN program of MIUR and by GNSAGA (Italy).

(C)2010 American Mathematical Society Reverts to public domain 28 years from publication 
Hence giving bounds for the absolute conditions automatically implies bounds on the relative one; moreover, one has that

$$
\begin{aligned}
& \mu_{1}^{[0]}=\text { first positive eigenvalue on functions for the Neumann condition, } \\
& \lambda_{1}^{[0]}=\mu_{1}^{[n]}=\text { first (positive) eigenvalue on functions for the Dirichlet condition. }
\end{aligned}
$$

In fact the absolute (resp. relative) boundary conditions on $p$-forms generalize the Neumann (resp. Dirichlet) conditions for functions. Moreover, the vector space of harmonic $p$-forms satisfying the absolute (resp. relative) conditions is isomorphic to the $p$-th de Rham absolute (resp. relative) cohomology space of $M$. General facts about the Laplacian on forms can be found in [15] and [2].

The scope of this paper is to give upper and lower bounds of the first positive eigenvalue $\mu_{1}^{[p]}$ when the manifold is a convex domain in the Euclidean space $\mathbf{R}^{n}$; see Theorems 1.1, 3.1 and 3.2 below. A lower bound of $\mu_{1}^{[p]}$ for Riemannian manifolds whose boundary has suitable degree of convexity is given in [7. For this and other results on the Hodge Laplacian on manifolds with non-empty boundary, see also the survey paper [8. Recent extrinsic estimates for submanifolds can be found in [13.

1.2. The main estimate. Now let $\Omega$ be a convex Euclidean domain of dimension $n$. It has been proven in Theorem 2.6 of [7] that the sequence of the first eigenvalues $\mu_{1}^{[p]}$ is non-decreasing with respect to the degree $p$; that is,

$$
\mu_{1}^{[0]}=\mu_{1}^{[1]} \leq \mu_{1}^{[2]} \leq \cdots \leq \mu_{1}^{[n]} .
$$

A brief comment on the equality $\mu_{1}^{[0]}=\mu_{1}^{[1]}$ : by differentiating eigenfunctions, one verifies the inequality $\mu_{1}^{[0]} \geq \mu_{1}^{[1]}$ (for an arbitrary manifold); however, equality does hold under the convexity assumption. This fact somehow shows that for a convex Euclidean domain the significant (first) eigenvalues are $\mu_{1}^{[1]}, \mu_{1}^{[2]}, \ldots, \mu_{1}^{[n]}$ which, by abuse of language, will also be called the "fundamental tones" of $\Omega$.

Our estimates show the following geometric property of the eigenvalues: up to constants (depending only on the degree and the dimension) the " $p$-th fundamental wavelength" $\frac{1}{\sqrt{\mu_{1}^{[p]}}}$ is equivalent to the $p$-th longest principal axis of $\Omega$, defined here as that of the unique ellipsoid of maximal volume included in $\Omega$.

Before giving the precise statement, let us also remark that the bounds in this paper could be seen as a generalization to $p$-forms of the following classical bounds for the Laplacian on functions, namely, the Payne-Weinberger inequality [12], valid for convex domains,

$$
\mu_{1}^{[0]}(\Omega) \geq \frac{\pi^{2}}{\operatorname{diam}(\Omega)^{2}}
$$

and the inequality due to Hersch [9] (later generalized by Li and Yau [11]), which is valid more generally when the mean curvature of $\partial \Omega$ is everywhere non-negative:

$$
\mu_{1}^{[n]}(\Omega)=\lambda_{1}^{[0]}(\Omega) \geq \frac{\pi^{2}}{4 R(\Omega)^{2}},
$$

where $R(\Omega)$ is the inner radius of $\Omega$ (that is, the radius of a largest ball included in $\Omega$ ).

We remark that the above inequalities have been extended by Li and Yau to Riemannian manifolds with non-negative Ricci curvature. From (1.2)-(1.4) and the 
monotonicity of the first Dirichlet eigenvalue (that is, $\mu_{1}^{[n]}$ ) with respect to inclusion we get, for all degrees $p$,

$$
\frac{\pi^{2}}{\operatorname{diam}(\Omega)^{2}} \leq \mu_{1}^{[p]}(\Omega) \leq \frac{\lambda_{1}^{[0]}\left(B^{n}\right)}{R(\Omega)^{2}}
$$

where $\lambda_{1}^{[0]}\left(B^{n}\right)$ is the first Dirichlet eigenvalue of the unit ball in $\mathbf{R}^{n}$. In [5], Pierre Guerini proves the lower bound:

$$
\mu_{1}^{[p]}(\Omega) \geq \frac{\max \{p(n-p), n-1\}}{n e^{3}} \cdot \frac{1}{\operatorname{diam}(\Omega)^{2}},
$$

which in many cases improves the one in (1.5).

Now, looking at the monotonicity property (1.2), one expects that it would be possible to improve the lower bounds in (1.5) and (1.6) by showing that

$$
\mu_{1}^{[p]}(\Omega) \geq \frac{a_{n, p}}{D_{p}(\Omega)^{2}}
$$

for a positive constant $a_{n, p}$ depending only on the degree and the dimension and for a non-increasing sequence of geometric invariants $D_{p}(\Omega)$, depending on the degree $p$, reducing to (half) the diameter when $p=1$ and to the inner radius when $p=n$.

Our main estimate, Theorem 1.1 below, states that this is in fact possible; moreover, one also has an upper bound of the same type.

Let us now define the invariants $D_{p}(\Omega)$. If $\Omega$ is a true ellipsoid, then $D_{p}$ is just the $p$-th longest principal axis of $\Omega$. If $\Omega$ is an arbitrary convex body, then $D_{p}$ will be the $p$-th longest principal axis of the ellipsoid $\mathcal{E}$ of maximal volume included in $\Omega$. By a well-known theorem in convex geometry, due to Fritz John (see [10] or also [1]), $\mathcal{E}$ is unique, and moreover,

$$
\mathcal{E} \subseteq \Omega \subseteq n \mathcal{E}
$$

where the homothety is taken with respect to the center of $\mathcal{E}$. In other words, if

$$
\gamma(\Omega)=\inf \{t \geq 1: \Omega \subseteq t \mathcal{E}\},
$$

then $\gamma(\Omega) \leq n$ (and actually $\gamma(\Omega) \leq \sqrt{n}$, provided that $\Omega$ is centrally symmetric).

$\mathcal{E}$ is known in the literature as the John ellipsoid of $\Omega$.

Let us now state the main bound in precise terms. We state it for the degree $p$ in the range $2, \ldots, n-1$, but the type of the bound also holds for $p=1$ and $p=n$ : these cases correspond in fact to the Laplacian on functions, the constants being given by the inequalities (1.3), (1.4) just mentioned and by an upper estimate due to Cheng (however, see Remark 1.2 below).

Theorem 1.1. Let $\Omega$ be a convex body in $\mathbf{R}^{n}, n \geq 3$, and $\mathcal{E}$ the (unique) ellipsoid of maximal volume included in $\Omega$, with principal axes: $D_{1}(\mathcal{E}) \geq D_{2}(\mathcal{E}) \geq \cdots \geq D_{n}(\mathcal{E})$. Then, for all $p=2, \ldots, n-1$ one has

$$
\frac{a_{n, p}}{D_{p}(\mathcal{E})^{2}}<\mu_{1}^{[p]}(\Omega)<\frac{a_{n, p}^{\prime}}{D_{p}(\mathcal{E})^{2}}
$$

where

$$
a_{n, p}=\frac{4}{n^{2} \cdot\left(\begin{array}{c}
n \\
p-1
\end{array}\right)}, \quad a_{n, p}^{\prime}=4 p(n+2) n^{n}
$$


We will actually prove the theorem with the following constants:

$$
a_{n, p}=\frac{4}{\gamma(\Omega)^{2} \cdot\left(\begin{array}{c}
n \\
p-1
\end{array}\right)}, \quad a_{n, p}^{\prime}=4 p(n+2) \gamma(\Omega)^{n},
$$

which improve the previous ones when an estimate of $\gamma(\Omega)$, better than $\gamma(\Omega) \leq n$, is available (that is, when $\Omega$ is close to being a true ellipsoid).

Remark 1.2. For completeness, we give the constants for $p=1$ and $p=n$. First observe that, as $\mathcal{E} \subseteq \Omega \subseteq n \mathcal{E}$, one has

$$
D_{1}(\mathcal{E}) \leq \operatorname{diam}(\Omega) \leq n D_{1}(\mathcal{E}) \quad \text { and } \quad D_{n}(\mathcal{E}) \leq 2 R(\Omega) \leq n D_{n}(\mathcal{E}) .
$$

1) The lower bound for $p=1$ is just the Payne-Weinberger inequality; therefore $a_{n, 1}=\frac{\pi^{2}}{n^{2}}$.

2) The upper bound for $p=1$ can be obtained by the following estimate of Cheng [3], valid for Riemannian manifolds with non-negative Ricci curvature (it also holds for convex Euclidean domains thanks to a doubling argument):

$$
\mu_{1}^{[0]} \leq \frac{n \pi^{2}}{\operatorname{diam}(\Omega)^{2}}, \quad \text { hence } \quad a_{n, 1}^{\prime}=n \pi^{2} .
$$

3) From the Hersch inequality (1.4) and the domain monotonicity of $\mu_{1}^{[n]}$ one finally has

$$
a_{n, n}=\frac{\pi^{2}}{n^{2}} \quad \text { and } \quad a_{n, n}^{\prime}=4 \lambda_{1}^{[0]}\left(B^{n}\right)
$$

Remark 1.3. The constants $a_{n, p}$ and $a_{n, p}^{\prime}$ are not sharp, as the strict inequality suggests, and we believe that they can be (perhaps significantly) improved. However, Theorem 1.1 is geometrically sharp in the sense that the invariant $D_{p}(\mathcal{E})$ determines the correct asymptotic behavior of the eigenvalue $\mu_{1}^{[p]}(\Omega)$ :

$$
\begin{aligned}
& \mu_{1}^{[p]}(\Omega) \rightarrow 0 \Longleftrightarrow D_{p}(\mathcal{E}) \rightarrow \infty \quad \text { and } \\
& \mu_{1}^{[p]}(\Omega) \rightarrow \infty \Longleftrightarrow D_{p}(\mathcal{E}) \rightarrow 0 .
\end{aligned}
$$

For example, if $\Omega$ "collapses", that is, it is contained in a smaller and smaller tubular neighborhood of an $m$-dimensional subspace of $\mathbf{R}^{n}$, then $D_{m+1}(\mathcal{E}) \rightarrow 0$ (and $D_{p}(\mathcal{E}) \rightarrow 0$ for all $\left.p \geq m+1\right)$. In that case one gets $\mu_{1}^{[p]}(\Omega) \rightarrow \infty$ for all $p \geq m+1$. Vice versa, one can detect collapsing (and the dimension of the subspace on which it takes place) just by counting the number of fundamental tones which diverge to infinity (this fact was conjectured by P. Guerini in his doctoral thesis). Note that the collapsing is controlled by explicit constants.

Therefore, Theorem 1.1 improves (1.5) and (1.6) when $D_{1}(\mathcal{E}) / D_{p}(\mathcal{E})$ is sufficiently large. It also improves (1.2), because the ratio $\mu_{1}^{[p+1]} / \mu_{1}^{[p]}$ is bounded above and below by a constant times the ratio $D_{p}(\mathcal{E})^{2} / D_{p+1}(\mathcal{E})^{2}$, which can be arbitrarily large.

1.3. Scheme of the proof. The proof of Theorem 1.1 will be given in Sections 3, 4 and 5. The upper (resp. lower) bound is given in terms of ellipsoids contained in (resp. containing) $\Omega$. John's theorem is then used to relate the two bounds. It is possible to estimate $\mu_{1}^{[p]}$ in terms of other invariants (equivalent to $D_{p}(\mathcal{E})$ up to constants); for example, the $p$-th longest side of a box containing $\Omega$ (see the remark 
in Section 3). In the next section we point out some easy consequences which, perhaps, are worth mentioning.

\section{Further REMARKS AND CONSEQUENCES}

From now on $a_{n, p}, a_{n, p}^{\prime}$ will refer to the constants in Theorem 1.1.

2.1. Spectral geometry. The bounds of Theorem 1.1 are equivalent to the fact that, if $\Omega$ is a convex body and $D_{p}(\mathcal{E})$ is the $p$-th longest principal axis of the ellipsoid of maximal volume included in $\Omega$, then

$$
\sqrt{\frac{a_{n, p}}{\mu_{1}^{[p]}(\Omega)}}<D_{p}(\mathcal{E})<\sqrt{\frac{a_{n, p}^{\prime}}{\mu_{1}^{[p]}(\Omega)}} .
$$

Hence if one can hear the fundamental tones of $\Omega$, then one can roughly guess the shape of $\Omega$, loosely interpreting the sequence of fundamental wavelengths; that is, $\frac{1}{\sqrt{\mu_{1}^{[1]}}}, \ldots, \frac{1}{\sqrt{\mu_{1}^{[n]}}}$ as the "principal axes of $\Omega$ ". In fact, let $\mathcal{E}_{\text {spec }}^{-}$be the ellipsoid of principal axes,

$$
D_{p}\left(\mathcal{E}_{\text {spec }}^{-}\right)=\sqrt{\frac{a_{n, p}}{\mu_{1}^{[p]}(\Omega)}}, \quad p=1, \ldots, n,
$$

and let $\mathcal{E}_{\text {spec }}^{+}$be the ellipsoid of principal axes,

$$
D_{p}\left(\mathcal{E}_{\text {spec }}^{+}\right)=\sqrt{\frac{a_{n, p}^{\prime}}{\mu_{1}^{[p]}(\Omega)}}, \quad p=1, \ldots, n .
$$

Then (2.1) says that $\mathcal{E}_{\text {spec }}^{-} \subset \mathcal{E} \subset \mathcal{E}_{\text {spec }}^{+}$. Recalling that $\mathcal{E} \subseteq \Omega \subseteq n \mathcal{E}$, one finally obtains:

Corollary 2.1. For all convex bodies $\Omega$ in $\mathbf{R}^{n}$ :

$$
\mathcal{E}_{\text {spec }}^{-} \subset \Omega \subset n \mathcal{E}_{\text {spec }}^{+} \text {. }
$$

2.2. Products of eigenvalues and the volume of cross-sections. For $p=$ $1, \ldots, n$ denote by vol ${ }^{[p]}(\Omega)$ the largest volume of a $p$-dimensional section of $\Omega$; that is,

$$
\operatorname{vol}^{[p]}(\Omega)=\sup \{\operatorname{vol}(\Sigma): \Sigma=\pi \cap \Omega, \pi \text { is a } p \text {-dimensional plane }\} .
$$

Note that $\mathrm{vol}^{[1]}$ is just the diameter and $\mathrm{vol}^{[n]}$ is the usual volume. Then:

Corollary 2.2. Let $\Omega$ be a convex body and $p=1, \ldots, n$. Then:

$$
\frac{c_{n, p}}{\sqrt{\mu_{1}^{[1]} \cdots \mu_{1}^{[p]}}}<\operatorname{vol}^{[p]}(\Omega)<\frac{c_{n, p}^{\prime}}{\sqrt{\mu_{1}^{[1]} \cdots \mu_{1}^{[p]}}},
$$

where

$$
c_{n, p}=2^{-p} \operatorname{vol}\left(B^{p}\right) \sqrt{\Pi_{k=1}^{k=p} a_{n, k}}, \quad c_{n, p}^{\prime}=\left(\frac{n}{2}\right)^{p} \operatorname{vol}\left(B^{p}\right) \sqrt{\Pi_{k=1}^{k=p} a_{n, k}^{\prime}}
$$

and $B^{p}$ is the p-dimensional unit ball. 
Proof. Since $\mathcal{E} \subseteq \Omega \subseteq n \mathcal{E}$ we obtain

$$
\operatorname{vol}^{[p]}(\mathcal{E}) \leq \operatorname{vol}^{[p]}(\Omega) \leq n^{p} \operatorname{vol}^{[p]}(\mathcal{E})
$$

We observe that

$$
\operatorname{vol}^{[p]}(\mathcal{E})=2^{-p} \operatorname{vol}\left(B^{p}\right) D_{1}(\mathcal{E}) \cdots D_{p}(\mathcal{E})
$$

since the $p$-dimensional section of $\mathcal{E}$ with largest volume is the one spanned by the longest $p$ principal axes. We now apply estimate (2.1).

Obviously the corollary is equivalent to an upper and lower estimate of the product $\mu_{1}^{[1]} \cdots \mu_{1}^{[p]}$ by the invariant $\operatorname{vol}^{[p]}(\Omega)^{-2}$; in particular, recalling that $\mu_{1}^{[k]}$ increases with $k$, one gets the (weaker) estimate:

Corollary 2.3. For all convex bodies $\Omega$ and $p=1, \ldots, n$,

$$
\mu_{1}^{[p]}(\Omega)>\left(\frac{c_{n, p}}{\operatorname{vol}^{[p]}(\Omega)}\right)^{2 / p} .
$$

2.3. Weak monotonicity. It is well known that the first Dirichlet eigenvalue is monotonic with respect to inclusion: if $\Omega \subseteq \Omega^{\prime}$, then

$$
\mu_{1}^{[n]}(\Omega) \geq \mu_{1}^{[n]}\left(\Omega^{\prime}\right)
$$

This fact fails to hold in degrees different from $n$ because, for each fixed degree $p<n$, there exists a family of domains $\Omega_{\epsilon}, \epsilon>0$, all contained in a ball of fixed radius and such that $\lim _{\epsilon \rightarrow 0} \mu_{1}^{[p]}\left(\Omega_{\epsilon}\right)=0$. These examples are due to P. Guerini and can be found in Theorem 2.1 of $\left[\underline{6}\right.$. However, the domains $\Omega_{\epsilon}$ are not convex.

Using the lower bound of Theorem 3.2(a) we can prove the following weak monotonicity principle in the convex case.

Corollary 2.4. Let $\Omega$ and $\Omega^{\prime}$ be convex bodies in $\mathbf{R}^{n}$, with $\Omega \subseteq \Omega^{\prime}$. Then for all $p<n$,

$$
\mu_{1}^{[p]}(\Omega)>b_{n, p} \mu_{1}^{[p]}\left(\Omega^{\prime}\right)
$$

where $b_{n, p}=\frac{a_{n, p}}{a_{n, p}^{\prime}}$.

Proof. Let $\mathcal{E}^{\prime}$ be the ellipsoid of maximal volume included in $\Omega^{\prime}$. By Theorem 1.1

$$
\mu_{1}^{[p]}\left(\Omega^{\prime}\right)<\frac{a_{n, p}^{\prime}}{D_{p}\left(\mathcal{E}^{\prime}\right)^{2}} .
$$

Now the ellipsoid $n \mathcal{E}^{\prime}$ contains $\Omega^{\prime}$; hence it also contains $\Omega$. By Theorem 3.2(a) one has

$$
\mu_{1}^{[p]}(\Omega)>\frac{a_{n, p}}{D_{p}\left(\mathcal{E}^{\prime}\right)^{2}} .
$$

The two inequalities give the assertion.

\section{THE UPPER AND LOWER BOUNDS}

The proof of Theorem 1.1 follows from two independent upper and lower estimates. We start from the upper bound, which is stated for any Euclidean domain and is given in terms of any ellipsoid contained in the domain. In what follows, $\mu_{1}^{[p]^{\prime}}$ (resp. $\mu_{m}^{[p]^{\prime}}$ ) denotes the first (resp. $m$-th) eigenvalue of the Laplacian restricted to the subspace of exact $p$-forms (see Section 4 for the relevant definitions; however, one has $\left.\mu_{m}^{[p]} \leq \mu_{m}^{[p]^{\prime}}\right)$. 
Theorem 3.1. (a) Let $\Omega$ be an arbitrary domain of $\mathbf{R}^{n}$ and $\mathcal{E}_{-}$any ellipsoid contained in $\Omega$ and having principal axes $D_{1}\left(\mathcal{E}_{-}\right) \geq D_{2}\left(\mathcal{E}_{-}\right) \geq \cdots \geq D_{n}\left(\mathcal{E}_{-}\right)$. Then, for all $p \geq 1$,

$$
\mu_{1}^{[p]^{\prime}}(\Omega)<4 p(n+2) \cdot \frac{\operatorname{vol}(\Omega)}{\operatorname{vol}\left(\mathcal{E}_{-}\right)} \cdot \frac{1}{D_{p}\left(\mathcal{E}_{-}\right)^{2}} .
$$

More generally, fix $h=p, \ldots, n$ and let $m=\left(\begin{array}{l}h \\ p\end{array}\right)$. Then

$$
\mu_{m}^{[p]^{\prime}}(\Omega)<4 p(n+2) \cdot \frac{\operatorname{vol}(\Omega)}{\operatorname{vol}\left(\mathcal{E}_{-}\right)} \cdot \frac{1}{D_{h}\left(\mathcal{E}_{-}\right)^{2}} .
$$

(b) If $\Omega$ is convex and $\mathcal{E}$ is the ellipsoid of maximal volume contained in $\Omega$, then

$$
\mu_{1}^{[p]^{\prime}}(\Omega)<4 p(n+2) \gamma(\Omega)^{n} \cdot \frac{1}{D_{p}(\mathcal{E})^{2}},
$$

where $\gamma(\Omega)=\inf \{t \geq 1: \Omega \subseteq t \mathcal{E}\}$. If $h=p, \ldots, n$ and $m=\left(\begin{array}{l}h \\ p\end{array}\right)$, then

$$
\mu_{m}^{[p]^{\prime}}(\Omega)<4 p(n+2) \gamma(\Omega)^{n} \cdot \frac{1}{D_{h}(\mathcal{E})^{2}} .
$$

The proof of the upper bound is given in the next section.

The lower bound is given in terms of any ellipsoid containing $\Omega$.

Theorem 3.2. (a) Let $\Omega$ be a convex body in $\mathbf{R}^{n}$ and $\mathcal{E}_{+}$any ellipsoid containing $\Omega$ with principal axes $D_{1}\left(\mathcal{E}_{+}\right) \geq D_{2}\left(\mathcal{E}_{+}\right) \geq \cdots \geq D_{n}\left(\mathcal{E}_{+}\right)$. Then, for all $p \geq 2$,

$$
\mu_{1}^{[p]}(\Omega)>4\left(\begin{array}{c}
n \\
p-1
\end{array}\right)^{-1} \cdot \frac{1}{D_{p}\left(\mathcal{E}_{+}\right)^{2}} .
$$

(b) If $\mathcal{E}$ is the ellipsoid of maximal volume contained in $\Omega$, then

$$
\mu_{1}^{[p]}(\Omega)>\frac{4}{\gamma(\Omega)^{2}\left(\begin{array}{c}
n \\
p-1
\end{array}\right)} \cdot \frac{1}{D_{p}(\mathcal{E})^{2}} .
$$

The proof of the lower bound will be given in Section 5 .

It is clear that parts (b) of the above two theorems prove Theorem 1.1 with the constants as in (1.10).

Remark 3.3. Instead of ellipsoids one can consider boxes. A box in $\mathbf{R}^{n}$ is a product of intervals: $\mathcal{B}=\left[0, b_{1}\right] \times \cdots \times\left[0, b_{n}\right]$, where we assume $b_{1} \geq \cdots \geq b_{n}>0$. Let us set $b_{p}(\mathcal{B})=b_{p}($ which is the $p$-th longest side of $\mathcal{B})$ and let

$$
b_{p}(\Omega)=\inf \left\{b_{p}(\mathcal{B}): \mathcal{B} \text { is a box containing } \Omega\right\} .
$$

We now verify that, for a convex body $\Omega$,

$$
\frac{4}{n\left(\begin{array}{c}
n \\
p-1
\end{array}\right)} \cdot \frac{1}{b_{p}(\Omega)^{2}} \leq \mu_{1}^{[p]}(\Omega) \leq \frac{n^{2} a_{n, p}^{\prime}}{b_{p}(\Omega)^{2}} .
$$

In fact, let $\mathcal{B}$ be the box with $b_{p}(\mathcal{B})=D_{p}(\mathcal{E})$. Then $\mathcal{B}$ contains $\mathcal{E}$ and by John's theorem we see that $D_{p}(\mathcal{E}) \geq \frac{1}{n} b_{p}(\Omega)$. Hence Theorem 1.1 gives the upper bound in (3.2). For the lower bound, let $\mathcal{B}$ be any box containing $\Omega$ and $\hat{\mathcal{E}}$ the ellipsoid of maximal volume included in $\mathcal{B}$. Clearly $D_{p}(\hat{\mathcal{E}})=b_{p}(\mathcal{B})$. By John's theorem the 
ellipsoid $\sqrt{n} \hat{\mathcal{E}}$ contains $\mathcal{B}$ and hence also $\Omega$, and we can apply Theorem 3.2(a) to it. As $D_{p}(\sqrt{n} \hat{\mathcal{E}})=\sqrt{n} b_{p}(\mathcal{B})$ we get

$$
\mu_{1}^{[p]}(\Omega) \geq \frac{4}{n\left(\begin{array}{c}
n \\
p-1
\end{array}\right)} \frac{1}{b_{p}(\mathcal{B})^{2}}
$$

which gives the lower bound because $\mathcal{B}$ was an arbitrary box containing $\Omega$.

\section{Proof of THE UPPER BOUND}

We start by recalling some facts concerning the Hodge Laplacian. A basic tool is the Hodge (or Hodge-Morrey) orthogonal decomposition of the space $\Lambda^{p}$ of $p$-forms on a manifold with boundary (see for example [14, Theorem 2.4.2):

$$
\Lambda^{p}=\delta \Lambda_{A}^{p+1} \oplus d \Lambda_{R}^{p-1} \oplus \mathcal{H}^{p},
$$

where

$$
\begin{aligned}
& \Lambda_{A}^{p}=\left\{\omega \in \Lambda^{p}: i_{N} \omega=0 \text { on } \partial \Omega\right\}, \\
& \Lambda_{R}^{p}=\left\{\omega \in \Lambda^{p}: J_{\partial \Omega} \omega=0\right\}, \\
& \mathcal{H}^{p}=\left\{\omega \in \Lambda^{p}: d \omega=\delta \omega=0\right\} .
\end{aligned}
$$

The space $\mathcal{H}^{p}$ is infinite dimensional, but its subspace $\mathcal{H}_{A}^{p}=\mathcal{H}^{p} \cap \Lambda_{A}^{p}$ is finite dimensional and isomorphic to the $p$-th de Rham absolute cohomology space of $\Omega$. It consists of all harmonic forms satisfying the absolute boundary conditions.

We will use the following easy consequence of 4.1). We say that a form $\omega$ is tangential if $i_{N} \omega=0$ on $\partial \Omega$.

Lemma 4.1. Let $\omega$ be a co-closed, tangential $p$-form on the domain $\Omega$ with $p=$ $1, \ldots, n-1$. Assume that $\mathcal{H}_{A}^{p}(\Omega)=0$ (this is true if $\Omega$ is convex). Then $\omega \in \delta \Lambda_{A}^{p+1}$; that is, $\omega$ is the co-differential of a tangential $(p+1)$-form.

Proof. By the Hodge-Morrey decomposition (4.1)

$$
\omega=\delta \xi+d \phi+h,
$$

with $i_{N} \xi=0$ and $d h=\delta h=0$. Now the scalar product of $\omega$ and $d \phi$ is zero by the Stokes formula, so that $d \phi=0$. As $\omega$ is tangential one also has $i_{N} h=0$, so $h$ is a cohomology class in $\mathcal{H}_{A}^{p}(\Omega)$, which is zero by assumption. Hence $\omega=\delta \xi$ with $\xi$ tangential.

As $\Delta$ commutes with both $d$ and $\delta$, one sees that

$$
\mu_{1}^{[p]}=\min \left\{\mu_{1}^{[p]^{\prime}}, \mu_{1}^{[p]^{\prime \prime}}\right\},
$$

where $\mu_{1}^{[p]^{\prime}}$ (resp. $\mu_{1}^{[p]^{\prime \prime}}$ ) is the first positive eigenvalue of $\Delta$ when restricted to the subspace of exact (resp. co-exact) forms. By differentiating eigenforms it follows that

$$
\mu_{1}^{[p-1]^{\prime \prime}}=\mu_{1}^{[p]^{\prime}} .
$$

Any exact $p$-form $\omega$ on $\Omega$ admits a canonical primitive. This is, by definition, the unique primitive $\theta$ of $\omega$ which belongs to $\delta \Lambda_{A}^{p}$. Hence $\theta$ satisfies the conditions

$$
d \theta=\omega, \theta=\delta \xi \quad \text { for some } p \text {-form } \xi \text { such that } i_{N} \xi=0 \text { on } \partial \Omega \text {. }
$$

If $\omega$ is an exact 1 -form its canonical primitive is the unique primitive $f$ which integrates to zero on the domain. The canonical primitive has the least $L^{2}$-norm among all primitives of $\omega$; it obviously depends on $\Omega$ and will be denoted by $\theta_{[\omega, \Omega]}$. 
The first positive co-exact eigenvalue satisfies the min-max principle:

$$
\mu_{1}^{[p]^{\prime \prime}}=\inf \left\{\frac{\int_{\Omega}\|d \theta\|^{2}}{\int_{\Omega}\|\theta\|^{2}}: \theta \in \delta \Lambda_{A}^{p+1}-\{0\}\right\} .
$$

From this, we can finally state the variational principle which will be used for the upper bound.

Lemma 4.2. Let $\Omega$ be an arbitrary domain and $\omega$ an exact $p$-form. Then

$$
\mu_{1}^{[p]^{\prime}}(\Omega) \leq \frac{\int_{\Omega}\|\omega\|^{2}}{\int_{\Omega}\left\|\theta_{[\omega, \Omega]}\right\|^{2}},
$$

where $\theta_{[\omega, \Omega]}$ is the canonical primitive of $\omega$ in $\Omega$. In particular, if $\Omega^{\prime}$ is a subdomain of $\Omega$, then

$$
\mu_{1}^{[p]^{\prime}}(\Omega) \leq \frac{\int_{\Omega}\|\omega\|^{2}}{\int_{\Omega^{\prime}}\left\|\theta_{\left[\omega, \Omega^{\prime}\right]}\right\|^{2}},
$$

where $\theta_{\left[\omega, \Omega^{\prime}\right]}$ now denotes the canonical primitive of $\omega$ on $\Omega^{\prime}$.

Proof. Given $\omega$, we use its canonical primitive $\theta_{[\omega, \Omega]} \in \delta \Lambda_{A}^{p}$ as a test-form for the eigenvalue $\mu_{1}^{[p-1]^{\prime \prime}}=\mu_{1}^{[p]^{\prime}}$. The first assertion now follows immediately from the min-max principle (4.5). The second statement follows from the first and from the fact that $\theta_{\left[\omega, \Omega^{\prime}\right]}$ minimizes the $L^{2}$-norm among all primitives of $\omega$ on $\Omega^{\prime}$ :

$$
\int_{\Omega}\left\|\theta_{[\omega, \Omega]}\right\|^{2} \geq \int_{\Omega^{\prime}}\left\|\theta_{[\omega, \Omega]}\right\|^{2} \geq \int_{\Omega^{\prime}}\left\|\theta_{\left[\omega, \Omega^{\prime}\right]}\right\|^{2} .
$$

Proof of Theorem 3.1. Let $\Omega$ be an arbitrary domain of $\mathbf{R}^{n}$ and $\mathcal{E}_{-}$any ellipsoid contained in $\Omega$. We first show that, for all $p \geq 1$,

$$
\mu_{1}^{[p]^{\prime}}(\Omega)<4 p(n+2) \cdot \frac{\operatorname{vol}(\Omega)}{\operatorname{vol}\left(\mathcal{E}_{-}\right)} \cdot \frac{1}{D_{p}\left(\mathcal{E}_{-}\right)^{2}} .
$$

We fix coordinates so that the included ellipsoid $\mathcal{E}_{-}$is expressed by the inequality

$$
\alpha_{1} x_{1}^{2}+\cdots+\alpha_{n} x_{n}^{2} \leq 1
$$

where $\alpha_{k}=\frac{4}{D_{k}\left(\mathcal{E}_{-}\right)^{2}}$; by assumption, $\alpha_{1} \leq \cdots \leq \alpha_{n}$. We choose the exact form $\omega=d x_{1} \wedge \cdots \wedge d x_{p}$ as a test $p$-form. By Lemma 4.2

$$
\mu_{1}^{[p]^{\prime}}(\Omega)<\frac{\operatorname{vol}(\Omega)}{\int_{\mathcal{E}_{-}}\|\theta\|^{2}},
$$

where $\theta=\theta_{\left[\omega, \mathcal{E}_{-}\right]}$is the canonical primitive of $\omega$ on the ellipsoid $\mathcal{E}_{-}$(the strict inequality holds because $\omega$ is harmonic, hence certainly not an eigenform associated to $\left.\mu_{1}^{[p]^{\prime}}(\Omega)\right)$. It turns out that $\theta$ is explicitly computable. In fact, let $\nu$ be the vector field $\nu=\sum_{k=1}^{n} \alpha_{k} x_{k} \partial / \partial_{k}$. Then

$$
\theta=\frac{1}{\alpha} \cdot i_{\nu}\left(d x_{1} \wedge \cdots \wedge d x_{p}\right)
$$

where $\alpha=\sum_{k=1}^{p} \alpha_{k}$. Explicitly,

$$
\theta=\frac{1}{\alpha} \sum_{k=1}^{p}(-1)^{k+1} \alpha_{k} x_{k} d x_{1} \wedge \cdots \wedge d \hat{x}_{k} \wedge \cdots \wedge d x_{p}
$$


To verify this, first observe that $\theta$ is indeed a primitive of $\omega$; moreover, it is co-closed because $\delta$ anticommutes with interior multiplication and $\omega$ is parallel. As $i_{\nu} \theta=0$, we see that $\theta$ is tangential because $\nu$ is orthogonal to the boundary of $\mathcal{E}_{-}$at any point of it. Then $\theta \in \delta \Lambda_{A}^{p+1}$ by Lemma 4.1 .

From (4.8) one gets

$$
\int_{\mathcal{E}_{-}}\|\theta\|^{2}=\frac{1}{\alpha^{2}} \sum_{k=1}^{p} \alpha_{k}^{2} \int_{\mathcal{E}_{-}} x_{k}^{2}
$$

Direct computation gives

$$
\int_{\mathcal{E}_{-}} x_{k}^{2}=\frac{\operatorname{vol}\left(\mathcal{E}_{-}\right)}{(n+2) \alpha_{k}}
$$

hence

$$
\int_{\mathcal{E}_{-}}\|\theta\|^{2}=\frac{1}{\sum_{k=1}^{p} \alpha_{k}} \cdot \frac{\operatorname{vol}\left(\mathcal{E}_{-}\right)}{n+2} \geq \frac{\operatorname{vol}\left(\mathcal{E}_{-}\right)}{p(n+2) \alpha_{p}} .
$$

Inserted in (4.7), this completes the proof of (4.6).

For the higher eigenvalues, we use the min-max principle,

$$
\mu_{m}^{[p]^{\prime}}(\Omega)=\inf _{V^{m}} \sup \left\{\frac{\int_{\Omega}\|\omega\|^{2}}{\int_{\Omega}\left\|\theta_{[\omega, \Omega]}\right\|^{2}}: \omega \in V^{m}-\{0\}\right\},
$$

where $V^{m}$ ranges over all $m$-dimensional subspaces of $d \Lambda^{p-1}$. Let $h$ be an integer between $p$ and $n$. We take $V^{m}$ as the subspace generated by all parallel exact forms

$$
d x_{i_{1}} \wedge \cdots \wedge d x_{i_{p}}
$$

with $i_{1}<\cdots<i_{p} \leq h$. It has dimension $m=\left(\begin{array}{l}h \\ p\end{array}\right)$. Note that each $\omega \in V^{m}$ has constant pointwise norm, which we may assume to be equal to 1 . Hence

$$
\mu_{m}^{[p]^{\prime}}(\Omega)<\sup \left\{\frac{\operatorname{vol}(\Omega)}{\int_{\mathcal{E}_{-}}\left\|\theta_{\left[\omega, \mathcal{E}_{-}\right]}\right\|^{2}}: \omega \in V^{m},\|\omega\|=1\right\} .
$$

Again, the form $\theta_{\left[\omega, \mathcal{E}_{-}\right]}$is explicit; proceeding as in (4.10) we see that for all such $\omega$ one has

$$
\int_{\mathcal{E}_{-}}\left\|\theta_{\left[\omega, \mathcal{E}_{-}\right]}\right\|^{2} \geq \frac{\operatorname{vol}\left(\mathcal{E}_{-}\right)}{4 p(n+2)} D_{h}\left(\mathcal{E}_{-}\right)^{2}
$$

(we omit the details because they are straightforward). Hence

$$
\mu_{m}^{[p]^{\prime}}(\Omega)<4 p(n+2) \frac{\operatorname{vol}(\Omega)}{\operatorname{vol}\left(\mathcal{E}_{-}\right)} \cdot \frac{1}{D_{h}\left(\mathcal{E}_{-}\right)^{2}},
$$

as asserted.

Now assume that $\Omega$ is convex. If we choose $\mathcal{E}_{-}=\mathcal{E}$, the ellipsoid of maximal volume contained in $\Omega$, then $\Omega \subseteq \gamma(\Omega) \mathcal{E}$, and so $\frac{\operatorname{vol}(\Omega)}{\operatorname{vol}(\mathcal{E})} \leq \gamma(\Omega)^{n}$. Inserted in the inequalities (4.6) and (4.11), this gives part (b) of Theorem 3.1. 


\section{Proof of the Lower bound (Theorem 3.2)}

In what follows we assume that $\Omega$ is a convex body in $\mathbf{R}^{n}$ (unless otherwise stated). Recall that a $p$-form $\omega$ is said to be "tangential" if $i_{N} \omega=0$ on $\partial \Omega$. Fix an orthonormal family of parallel vector fields $V_{1}, \ldots, V_{p}$. The proof of the lower bound is based on two independent estimates of the $L^{2}$-norm of the smooth function $\omega\left(V_{1}, \ldots, V_{p}\right)$, where $\omega$ is a co-closed, tangential $p$-form. This will lead to a lower bound for the energy of co-closed tangential forms which is of independent interest and is the main step in the proof (see Lemma 5.3). The final conclusion is then obtained by applying the Bochner formula to a co-exact eigenform.

Denote by $V_{1} \wedge \cdots \wedge V_{p}$ the $p$-dimensional plane through the origin spanned by $V_{1}, \ldots, V_{p}$. The expression " $\Sigma$ is a section orthogonal to $V_{1} \wedge \cdots \wedge V_{p}$ " means that

$$
\Sigma=\pi \cap \Omega, \text { where } \pi \text { is a plane parallel to }\left(V_{1} \wedge \cdots \wedge V_{p}\right)^{\perp} .
$$

Clearly $\Sigma$ is a convex set in the plane $\pi$. For example, if a coordinate system has been fixed and $V_{k}$ is the $k$-th coordinate field $\partial / \partial x_{k}$, then such a section $\Sigma$ is described by the equations

$$
\Sigma=\left\{\left(x_{1}, \ldots, x_{n}\right) \in \Omega: x_{1}=\xi_{1}, \ldots, x_{p}=\xi_{p}\right\}
$$

for a suitable $\left(\xi_{1}, \ldots, \xi_{p}\right) \in \mathbf{R}^{p}$. We set

$$
d\left(V_{1} \wedge \cdots \wedge V_{p}\right)=\sup \left\{\operatorname{diam}(\Sigma): \Sigma \text { is a section orthogonal to } V_{1} \wedge \cdots \wedge V_{p}\right\}
$$

Lemma 5.1. Let $\omega$ be a co-closed, tangential $p$-form on the convex body $\Omega$ and let $V_{1}, \ldots, V_{p}$ be an orthonormal family of parallel vector fields and $f=\omega\left(V_{1}, \ldots, V_{p}\right)$. Then:

(a) The function $f$ has zero integral when restricted to any section $\Sigma$ orthogonal to $V_{1} \wedge \cdots \wedge V_{p}$.

(b) One has

$$
\int_{\Omega}\|d f\|^{2} \geq \frac{\pi^{2}}{d\left(V_{1} \wedge \cdots \wedge V_{p}\right)^{2}} \int_{\Omega} f^{2}
$$

Proof of (a). By Lemma 4.1 we can write $\omega=\delta \xi$ with $\xi$ tangential. Consider the 1-form $\hat{\xi}$ on $\Sigma$ defined by

$$
\hat{\xi}(X)=\xi\left(X, V_{1}, \ldots, V_{p}\right)
$$

We show that $\hat{\xi}$ is tangential (on $\partial \Sigma$ ) and $\delta \hat{\xi}=f$ on $\Sigma$; hence $f$ integrates to zero on $\Sigma$ by the Stokes formula. Fix an orthonormal parallel frame $\left(e_{1}, \ldots, e_{n-p}\right)$ on $\Sigma$ so that the vectors $\left(e_{1}, \ldots, e_{n-p}, V_{1}, \ldots, V_{p}\right)$ form an orthonormal parallel frame in $\mathbf{R}^{n}$. Then

$$
\begin{aligned}
\delta \hat{\xi} & =-\sum_{k=1}^{n-p} e_{k} \cdot \hat{\xi}\left(e_{k}\right) \\
& =-\sum_{k=1}^{n-p} e_{k} \cdot \xi\left(e_{k}, V_{1}, \ldots, V_{p}\right) \\
& =\delta \xi\left(V_{1}, \ldots, V_{p}\right) \\
& =f .
\end{aligned}
$$

It remains to show that $\hat{\xi}\left(N_{\Sigma}\right)=0$, where $N_{\Sigma}$ is a unit vector, normal to $\partial \Sigma$ at a given point $x \in \partial \Sigma$. Denote by $\bar{X}$ the orthogonal projection of the vector $X \in \mathbf{R}^{n}$ 
onto $T_{x} \partial \Omega$. Since $i_{N} \xi=0$ we see that

$$
\begin{aligned}
\hat{\xi}\left(N_{\Sigma}\right) & =\xi\left(N_{\Sigma}, V_{1}, \ldots, V_{p}\right) \\
& =\xi\left(\bar{N}_{\Sigma}, \bar{V}_{1}, \ldots, \bar{V}_{p}\right) .
\end{aligned}
$$

The $p+2$ vectors $V_{1}, \ldots, V_{p}, N, N_{\Sigma}$ are linearly dependent because they all belong to the orthogonal complement of the subspace $T_{x}(\partial \Sigma)$, which has dimension $n-p-1$. As $\bar{N}=0$, the vectors $\bar{V}_{1}, \ldots, \bar{V}_{p}, \bar{N}_{\Sigma}$ are dependent; hence $\xi\left(\bar{N}_{\Sigma}, \bar{V}_{1}, \ldots, \bar{V}_{p}\right)=0$ as asserted.

Proof of (b). By part (a) we can use $f$ (or more precisely $J_{\Sigma} f$ ) as a test-function for the eigenvalue $\mu_{1}^{[0]}(\Sigma)$; the Payne-Weinberger inequality (1.3) applied to $\Sigma$ (which is convex) gives

$$
\int_{\Sigma}\left\|J_{\Sigma} d f\right\|^{2} \geq \frac{\pi^{2}}{\operatorname{diam}(\Sigma)^{2}} \int_{\Sigma} f^{2}
$$

As $\left\|J_{\Sigma} d f\right\|^{2} \leq\|d f\|^{2}$ one then gets, recalling definition (5.1),

$$
\int_{\Sigma}\|d f\|^{2} \geq \frac{\pi^{2}}{d\left(V_{1} \wedge \cdots \wedge V_{p}\right)^{2}} \cdot \int_{\Sigma} f^{2}
$$

The assertion now follows by integrating the above inequality over all sections $\Sigma$ orthogonal to $V_{1} \wedge \cdots \wedge V_{p}$ (applying Fubini's theorem in the obvious way).

Lemma 5.2. Here $\Omega$ is an arbitrary domain of $\mathbf{R}^{n}$. Let $V_{1}, \ldots, V_{p}$ be an orthonormal family of parallel vector fields, $\omega$ a co-closed, tangential $p$-form on $\Omega$ and $f=\omega\left(V_{1}, \ldots, V_{p}\right)$. Then

$$
\left\{\int_{\Omega} f^{2}\right\}^{2} \leq \max _{\Omega}\left|\rho_{p}\right|^{2} \cdot \int_{\Omega}\|\omega\|^{2} \cdot \int_{\Omega}\|d f\|^{2},
$$

where $\rho_{p}$ is the distance (taken with sign) from the hyperplane through the origin orthogonal to $V_{p}$ (so that $\nabla \rho_{p}=V_{p}$ ).

Proof. Consider the 1-form $\eta$ defined by

$$
\eta(X)=\omega\left(V_{1}, \ldots, V_{p-1}, X\right),
$$

so that $f=i_{V_{p}} \eta$. As $\delta$ anticommutes with interior multiplication, one sees that $\eta$ is co-closed; moreover, $i_{N} \eta=0$ on $\partial \Omega$. Given a smooth function $g$ we observe the identity:

$$
\delta(g \eta)=-i_{\nabla g} \eta+g \delta \eta .
$$

Taking $g=\rho_{p}$ one has $\nabla g=V_{p}$; as $\delta \eta=0$ we can express $f$ as follows:

$$
f=-\delta\left(\rho_{p} \eta\right)
$$

Any form $\alpha$ obeys Stokes' formula:

$$
\int_{\Omega}\|\delta \alpha\|^{2}=\int_{\Omega}\langle\alpha, d \delta \alpha\rangle+\int_{\partial \Omega}\left\langle i_{N} \alpha, \delta \alpha\right\rangle .
$$

If $\alpha=\rho_{p} \eta$ we then conclude by (5.2) that

$$
\int_{\Omega} f^{2}=-\int_{\Omega} \rho_{p}\langle\eta, d f\rangle .
$$


By the Cauchy-Schwarz inequality, since $\|\eta\|^{2} \leq\|\omega\|^{2}$, we then get

$$
\begin{aligned}
\left\{\int_{\Omega} f^{2}\right\}^{2} & \leq \int_{\Omega}\left|\rho_{p}\right|^{2}\|\omega\|^{2} \cdot \int_{\Omega}\|d f\|^{2} \\
& \leq \max _{\Omega}\left|\rho_{p}\right|^{2} \cdot \int_{\Omega}\|\omega\|^{2} \cdot \int_{\Omega}\|d f\|^{2} .
\end{aligned}
$$

The following estimate on the energy is the final step in the proof of Theorem 3.2 .

Lemma 5.3. Let $\Omega$ be a convex body in $\mathbf{R}^{n}$ and $\mathcal{E}_{+}$any ellipsoid containing $\Omega$. Let $\omega$ be a co-closed, tangential $p$-form $(p=1, \ldots, n-1)$ on $\Omega$. Then

$$
\int_{\Omega}\|\nabla \omega\|^{2}>4\left(\begin{array}{l}
n \\
p
\end{array}\right)^{-1} \frac{1}{D_{p+1}\left(\mathcal{E}_{+}\right)^{2}} \int_{\Omega}\|\omega\|^{2}
$$

Proof. We can assume that $\omega$ has unit $L^{2}$-norm. Fix a coordinate system $\left(x_{1}, \ldots\right.$, $x_{n}$ ) so that the ellipsoid $\mathcal{E}_{+}$is expressed as

$$
\frac{x_{1}^{2}}{D_{1}^{2}}+\cdots+\frac{x_{n}^{2}}{D_{n}^{2}} \leq 1
$$

with $D_{k}=\frac{1}{2} D_{k}\left(\mathcal{E}_{+}\right)$being the $k$-th principal semi-axis of $\mathcal{E}_{+}$. By assumption $D_{1} \geq \cdots \geq \stackrel{2}{D_{n}}$.

From now on $V_{i}$ will be the $i$-th coordinate field $\frac{\partial}{\partial x_{i}}$, and we write for simplicity

$$
\omega_{i_{1} \ldots i_{p}}=\omega\left(V_{i_{1}}, \ldots, V_{i_{p}}\right), \quad P_{i_{1} \ldots i_{p}}=\int_{\Omega} \omega_{i_{1} \ldots i_{p}}^{2}
$$

so that

$$
\sum_{i_{1}<i_{2}<\cdots<i_{p}} P_{i_{1} \ldots i_{p}}=\int_{\Omega}\|\omega\|^{2}=1 .
$$

If $\Sigma=\pi \cap \Omega$ is any section by an $(n-p)$-dimensional plane $\pi$ orthogonal to $V_{1} \wedge \cdots \wedge V_{p}$, then, on $\Sigma$, the coordinate functions $x_{1}, \ldots, x_{p}$ are constant and $\Sigma$ is contained in the ellipsoid $\pi \cap \mathcal{E}_{+}$given by the inequality

$$
\frac{x_{p+1}^{2}}{D_{p+1}^{2}}+\cdots+\frac{x_{n}^{2}}{D_{n}^{2}} \leq 1
$$

This shows that $\operatorname{diam}(\Sigma) \leq 2 D_{p+1}$. If $f=\omega_{1 \ldots p}$, then by Lemma 5.1

$$
\int_{\Omega}\left\|d \omega_{1 \ldots p}\right\|^{2} \geq \frac{\pi^{2}}{4 D_{p+1}^{2}} \cdot \int_{\Omega}\left|\omega_{1 \ldots p}\right|^{2}=\frac{\pi^{2}}{4 D_{p+1}^{2}} P_{1 \ldots p} .
$$

On the other hand, given a multi-index $i_{1}<i_{2}<\cdots<i_{p}$ and letting $f=\omega_{i_{1} i_{2} \ldots i_{p}}$, Lemma 5.2 implies that

$$
\int_{\Omega}\left\|d \omega_{i_{1} \ldots i_{p}}\right\|^{2} \geq \frac{P_{i_{1} \ldots i_{p}}^{2}}{D_{i_{p}}^{2}}
$$


because $\max \left|\rho_{i_{p}}\right|^{2} \leq D_{i_{p}}^{2}$. Hence

$$
\begin{aligned}
\int_{\Omega}\|\nabla \omega\|^{2} & =\int_{\Omega}\left\|d \omega_{1 \ldots p}\right\|^{2}+\sum_{i_{1}<\cdots<i_{p}, i_{p} \geq p+1} \int_{\Omega}\left\|d \omega_{i_{1} \ldots i_{p}}\right\|^{2} \\
& \geq \frac{\pi^{2}}{4} \cdot \frac{P_{1 \ldots p}}{D_{p+1}^{2}}+\sum_{i_{1}<\cdots<i_{p}, i_{p} \geq p+1} \frac{P_{i_{1} \ldots i_{p}}^{2}}{D_{i_{p}}^{2}} \\
& \geq \frac{1}{D_{p+1}^{2}}\left[\frac{\pi^{2}}{4} P_{1 \ldots p}+\sum_{i_{1}<\cdots<i_{p}, i_{p} \geq p+1} P_{i_{1} \ldots i_{p}}^{2}\right] .
\end{aligned}
$$

Assume $P_{1 \ldots p}>0$. As $\frac{\pi^{2}}{4}>1$ and $P_{1 \ldots p} \geq P_{1 \ldots p}^{2}$ we obtain

$$
\int_{\Omega}\|\nabla \omega\|^{2}>\frac{1}{D_{p+1}^{2}} \cdot \sum_{i_{1}<\cdots<i_{p}} P_{i_{1} \ldots i_{p}}^{2} .
$$

By the Cauchy-Schwarz inequality

$$
\sum_{i_{1}<\cdots<i_{p}} P_{i_{1} \ldots i_{p}}^{2} \geq\left(\begin{array}{l}
n \\
p
\end{array}\right)^{-1}\left\{\sum_{i_{1}<\cdots<i_{p}} P_{i_{1} \ldots i_{p}}\right\}^{2}=\left(\begin{array}{c}
n \\
p
\end{array}\right)^{-1}
$$

which substituted in (5.9) gives

$$
\int_{\Omega}\|\nabla \omega\|^{2}>\left(\begin{array}{l}
n \\
p
\end{array}\right)^{-1} \cdot \frac{1}{D_{p+1}^{2}}=4\left(\begin{array}{l}
n \\
p
\end{array}\right)^{-1} \cdot \frac{1}{D_{p+1}(\mathcal{E})^{2}},
$$

as asserted. Note that if $P_{1 \ldots p}=0$ the constant $\left(\begin{array}{l}n \\ p\end{array}\right)^{-1}$ is replaced by the larger constant $\left(\left(\begin{array}{l}n \\ p\end{array}\right)-1\right)^{-1}$. Hence the strict inequality in (5.10) would hold as well.

We can now prove our main lower bound, Theorem 3.2. Let $\Omega$ be a convex body in $\mathbf{R}^{n}$ and $\mathcal{E}_{+}$an ellipsoid containing $\Omega$, with principal axes $D_{1}\left(\mathcal{E}_{+}\right) \geq D_{2}\left(\mathcal{E}_{+}\right) \geq$ $\cdots \geq D_{n}\left(\mathcal{E}_{+}\right)$. We have to prove that, for all $p \geq 2$,

$$
\mu_{1}^{[p]}(\Omega)>4\left(\begin{array}{c}
n \\
p-1
\end{array}\right)^{-1} \cdot \frac{1}{D_{p}\left(\mathcal{E}_{+}\right)^{2}} .
$$

Moreover, if $\mathcal{E}$ is the ellipsoid of maximal volume contained in $\Omega$, then

$$
\mu_{1}^{[p]}(\Omega)>\frac{4}{\gamma(\Omega)^{2}\left(\begin{array}{c}
n \\
p-1
\end{array}\right)} \cdot \frac{1}{D_{p}(\mathcal{E})^{2}} .
$$

Proof of (5.11). From the proof of Theorem 2.6 in $[7$ we know that, if $\Omega$ is convex, then $\mu_{1}^{[p]^{\prime}} \leq \mu_{1}^{[p]^{\prime \prime}}$ so that

$$
\mu_{1}^{[p]}=\mu_{1}^{[p]^{\prime}}=\mu_{1}^{[p-1]^{\prime \prime}} .
$$

For simplicity of notation we prove the assertion in degree $p+1$ : then let $\omega$ be an eigen $p$-form associated to $\mu_{1}^{[p+1]}=\mu_{1}^{[p]^{\prime \prime}}$. By definition, $\omega$ is co-exact and tangential, and we can (and will) assume that it has a unit $L^{2}$-norm.

The Bochner formula applied to the eigenform $\omega$ gives

$$
\mu_{1}^{[p+1]}\|\omega\|^{2}=\|\nabla \omega\|^{2}+\frac{1}{2} \Delta\left(\|\omega\|^{2}\right) .
$$


As $\omega$ satisfies the absolute boundary conditions and $\Omega$ is convex (so that the principal curvatures of $\partial \Omega$ are all non-negative), one has, by Lemma 4.10(b) in [7,

$$
\int_{\Omega} \Delta\left(\|\omega\|^{2}\right)=\int_{\partial \Omega} \frac{\partial\|\omega\|^{2}}{\partial N} \geq 0
$$

In fact, on the boundary, the normal derivative of $\|\omega\|^{2}$ is given by $2\left\langle S^{[p]} \omega, \omega\right\rangle$, where $S^{[p]}$ denotes the shape operator acting on $p$-forms; by the convexity assumption it is non-negative. Integrating (5.13) over $\Omega$ and taking into account (5.14) we finally obtain, by Lemma 5.3,

$$
\begin{aligned}
\mu_{1}^{[p+1]} & \geq \int_{\Omega}\|\nabla \omega\|^{2} \\
& >\left(\begin{array}{c}
n \\
p
\end{array}\right)^{-1} \cdot \frac{4}{D_{p+1}\left(\mathcal{E}_{+}\right)^{2}} .
\end{aligned}
$$

Replacing $p$ by $p-1$ we obtain (5.11).

Proof of (5.12). If $\mathcal{E}$ is the ellipsoid of maximal volume included in $\Omega$, then the ellipsoid $\mathcal{E}_{+}=\gamma(\Omega) \cdot \mathcal{E}$ contains $\Omega$. Applying (5.11) to $\mathcal{E}_{+}$we obtain (5.12) immediately.

\section{REFERENCES}

[1] K. Ball, Ellipsoids of maximal volume in convex bodies, Geom. Dedicata 4, no. 2 (1992) 241-250. MR.1153987 (93k:52006)

[2] I. Chavel, Eigenvalues in Riemannian Geometry (Appendix by J. Dodziuk), Academic Press Inc., 1984. MR768584 (86g:58140)

[3] S.Y. Cheng, Eigenvalue comparison theorems and its geometric applications, Math. Z., 143 (1975) 289-297. MR0378001 (51:14170)

[4] S. Gallot and D. Meyer, D'un résultat hilbertien à un principe de comparaison entre spectres. Applications, Ann. Scient. Ec. Norm. Sup., 4a sèrie, 21 (1988) 561-591. MR982334 (90k:58236)

[5] P. Guerini, Spectre du Laplacien de Hodge-de Rham: Estimées sur le variétés convexes, Bull. London Math. Soc., 36, no. 1 (2004) 88-94. MR2011982 (2004i:58055)

[6] P. Guerini, Prescription du spectre du Laplacien de Hodge-de Rham, Ann. Scient. Ec. Norm. Sup. 4a-sèrie, 37 (2004) 270-303. MR2061782 (2005k:58063)

[7] P. Guerini and A. Savo, Eigenvalue and gap estimates for the Laplacian acting on p-forms, Trans. Amer. Math. Soc. 356, no. 1 (2003) 319-344. MR2020035 (2005e:58049)

[8] P. Guerini and A. Savo, The Hodge Laplacian on manifolds with boundary, Séminaire de Théorie Spectrale et Géométrie (Univ. Grenoble I, Saint-Martin-d'Hères) 21, Année 20022003, 125-146. MR2052829 (2005i:58031)

[9] J. Hersch, Sur la frèquence fondamentale d'une membrane vibrante: Évaluations par defaut et principe de maximum, J. Math. Phy. Appl., XI, Fasc. 5 (1960) 387-413. MR0125319 (23:A2622)

[10] F. John, Extremum problems with inequalities as a subsidiary conditions, pp. 187-204 in: Studies and essays presented to R. Courant on his 60th birthday (January 8, 1948) Interscience, New York, 1948. MR0030135(10:719b)

[11] P. Li and S-T Yau, Estimates of eigenvalues of a compact Riemannian manifold, Proc. Symp. Pure Math., 36 (1980) 205-239. MR573435 (81i:58050)

[12] L. Payne and H.F. Weinberger, Lower bounds for vibration frequencies of elastically supported membranes and plates, J. Soc. Ind. Appl. Math., 5 (1957) 171-182. MR0092431 (19:1110c)

[13] A. Savo, On the first Hodge eigenvalue of isometric immersions, Proc. Amer. Math. Soc., 133, no. 2 (2005) 587-594. MR2093083(2005h:58053) 
[14] G. Schwarz, Hodge decomposition - a method to solve boundary value problems, Lecture Notes in Mathematics, Springer-Verlag, 1995. MR1367287 (96k:58222)

[15] M. E. Taylor, Partial Differential Equations, Basic Theory, Text in Applied Mathematics 115, Springer, 1996. MR.1395147(98b:35002a)

Dipartimento di Scienze di Base e Applicate per l'Ingegneria - Sezione di Matematica, Sapienza Università di Roma, Via Antonio Scarpa 14, 00161 Roma, Italy

E-mail address: savo@dmmm.uniroma1.it 